\title{
Application of Thyroid Peroxidase (TPO) and Hector Battifora Mesothelial-1 (HBME-1) immunohistochemical markers in the diagnosis of papillary thyroid carcinoma of the thyroid
}

\author{
Singh V. ${ }^{1}$, Kaur Bagga P. ${ }^{2 *}$, Singh B. ${ }^{3}$, Jaideep ${ }^{4}$ \\ DOI: https://doi.org/10.17511/ijmrr.2020.i01.17 \\ 1 Vasundhara Singh, Junior Resident, Department of Pathology, Government Medical College, Amritsar, Punjab, India. \\ 2* Permeet Kaur Bagga, Associate Professor, Department of Pathology, Government Medical College, Amritsar, Punjab, India. \\ 3 Bikramjeet Singh, Associate Professor, Department of Surgery, Government Medical College, Amritsar, Punjab, India. \\ 4 Jaideep, Assistant Professor, Department of Pathology, Government Medical College, Amritsar, Punjab, India.
}

Background: Thyroid cancer is the most common endocrine malignancy accounting for $>90 \%$ of malignancies of endocrine glands. The inter and intraobserver variation in the histomorphological diagnosis of Papillary Thyroid Carcinomas may sometimes pose a diagnostic difficulty. Application of IHC biomarkers may play an active or complementary role in their accurate classification. Aim: The present study was conducted to evaluate if HBME-1 and TPO immunohistochemical analysis can reliably differentiate papillary carcinomas from other thyroid lesions. Material and Methods: 50 cases of benign and malignant thyroid lesions were taken. Immunohistochemical staining for HBME1 and TPO was performed. HBME-1 and TPO score was interpreted as absent and positive. Medical records were retrieved and their clinical data, surgical treatment, and pathological findings were noted. Results: Out of 50 cases, 19 (73.1\%) cases were diagnosed PTC, 4 (15.4\%) cases were FTC, $3(11.5 \%)$ cases were of MTC and 24 cases of benign thyroid lesions. TPO expression was found positive in $91.7 \%$ of cases of Benign thyroid lesions. In malignant thyroid lesions, negative expression of TPO was seen in $63.16 \%, 0 \%$ and $33.33 \%$ of PTC, FCT, and MCT respectively. Conclusion: Testing for expression of HBME-1 has been shown to improve the diagnostic accuracy for thyroid malignant nodules. The combination of HBME-1, and TPO may contribute to an accurate diagnosis of papillary thyroid carcinoma.

Keywords: TPO; HBME-1, Papillary Thyroid Carcinoma, Immunohistochemistry

Corresponding Author

Permeet Kaur Bagga, Associate Professor, Department of Pathology, Government Medical College, Amritsar, Punjab, India.

Email: permeetbagga18@yahoo.co.in
How to Cite this Article

Singh V, Bagga PK, Singh B, Jaideep. Application of Thyroid Peroxidase (TPO) and Hector Battifora Mesothelial-1 (HBME-1) immunohistochemical markers in the diagnosis of papillary thyroid carcinoma of the thyroid. Int J Med Res Rev. 2020;8(1):110-117.

Available From

https://ijmrr.medresearch.in/index.php/ijmrr/article/

view/1111
To Browse

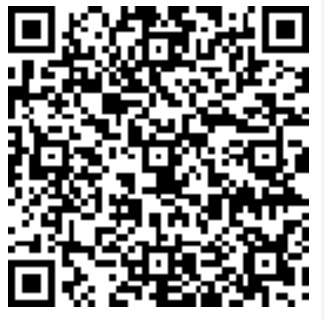

Manuscript Received 2020-01-22

Conflict of Interest No
Review Round 1 2020-02-02

Funding $\mathrm{Nil}$
Review Round 2 2020-02-07

Ethical Approval Yes
Review Round 3

Accepted 2020-02-11

Plagiarism X-checker $7 \%$

Note

(C) 2020 by Vasundhara Singh, Permeet Kaur Bagga, Bikramjeet Singh, Jaideep and Published by Siddharth Health Research and 


\section{Introduction}

Thyroid cancer is the most common endocrine malignancy accounting for $>90 \%$ of malignancies of endocrine glands [1] and Papillary carcinoma is the most common tumor of all the thyroid malignancies, constituting $80 \%$ of all thyroid malignancies [2]. Papillary thyroid carcinoma is defined as a malignant epithelial tumor showing evidence of follicular cell differentiation and is characterized by distinctive nuclear features [3]. Papillary thyroid carcinoma is usually discovered on routine examination as asymptomatic thyroid nodule that appears as a neck mass. In some instances, the mass may produce local symptoms. It is also one of the least aggressive cancer types and is slowgrowing [4]. Some tumors are well-circumscribed and even encapsulated; others infiltrate the adjacent parenchyma and have ill-defined margins. Historically, the identification of papillary carcinoma relied on the presence of papillary architecture. The currently accepted diagnosis is based on the nuclear features that include optical clearing, overlapping, nuclear grooves, and pseudo inclusions. The microscopic hallmarks of papillary neoplasm includes branching papillae having fibrovascular stalk covered by single to multiple layers of cuboidal epithelial cells, the nuclei contain finely dispersed chromatin, which imparts an optically clear or empty appearance, giving rise to ground glass or Orphan Annie eye nuclei, invagination of cytoplasm gives intranuclear inclusion appearance (pseudo inclusion), psammoma bodies (concentric calcified bodies). This tumor gains the maximum attention because of its increasing incidence, quick spread effectively through the lymphatic ducts, resulting frequently in recurrences, metastases and even death [5]. However, identification of these features remains, at times controversial and the distinction of papillary carcinoma from its histological mimickers such as follicular adenoma and follicular carcinoma may sometimes be difficult [6]. It is clear that some cases do raise controversy as being PTC or non-PTC. For example, follicular adenoma and follicular variant of PTC, when some of the nuclear diagnostic criteria for PTC are occasionally present. Unfortunately, such controversy exists between expert thyroid pathologists. Interobserver disagreement among pathologists is well documented 5 and some cases are unjustifiably over called a follicular variant of PTC. Immunohistochemistry (IHC) is the process whereby antibodies are used to detect proteins
(Antigens) in cells within a tissue section (for instance, thyroid tissue). Several diseases or disease subtypes may look alike or appear to have similar size cells under a microscope but have different behavior and necessary treatments. The best way to differentiate them is to detect specific molecules on these cells that act as markers. Immunohistochemistry (IHC) is a technique that uses antibodies (matching molecules) that can seek out, identify and attach themselves to these markers on cells. The antibodies themselves can be seen under the microscope, which helps the technician make a precise identification. Many Immunohistochemistry (IHC) markers have been described and validated for differentiating benign thyroid lesions from malignant ones and Follicular Variant of Papillary Carcinoma (FVPTC) from Follicular Carcinoma (FCa) or Follicular Adenoma (FA) [7]. In the present study, analysis of the IHC markers (TPO and HBME-1) was done to differentiate between benign and malignant surgically resected Thyroid lesions along with their utility in the identification of PTC.

\section{Material and Method}

Setting: This study was done at Government Medical College, Amritsar which is a Tertiary care center.

Type of study and sampling method: A retrospective review of the database of 50 patients diagnosed with thyroid lesions was taken.

Inclusion criteria: All specimens of thyroid tissue, diagnosed histologically as benign or malignant lesions of the thyroid, were included irrespective of age.

Exclusion criteria: All the patients with metastasis to the thyroid gland were excluded.

Data collection procedure and data analysis: Medical records of 50 patients with benign and malignant thyroid lesions who underwent surgery were retrieved and their clinical data, surgical treatment and histopathology reports along with their histopathology slides were reviewed and the respective histopathology blocks were retrieved for IHC procedure. HBME-1 and TPO markers were used and their scoring was done by using the IRS system.

Ethical consideration and permission: All procedures were done and the methods used in this study were in accordance with the ethical standard 
Of the institutional research committee.

IHC Staining Method: Tissue used for this study was obtained from the patients mentioned above at the Department of Pathology, Government Medical College, Amritsar. The tissues were embedded in paraffin and sliced into 3-4 $\mu \mathrm{m}$ sections, which was mounted on freshly prepared $0.01 \%$ poly-L-lysine coated slides. Slides were dried overnight at $370 \mathrm{C}$, dewaxed in xylene and hydrated. Endogenous peroxidase activity was blocked by adding freshly prepared $0.3 \% \mathrm{H}_{2} \mathrm{O} 2$ in methanol for 10 minutes, followed by three washings in PBS. Antigen retrieval was done as per the specification of the kit. Slides were then immersed in citrate buffer and put in the microwave oven for 2 cycles of 10 to 15 minutes at $850 \mathrm{C}$ and $1000 \mathrm{C}$ temperature respectively. The slides were bought to room temperature and immersed in PBS. Sections were washed in phosphate buffer saline and 2 drops of $3 \% \mathrm{H}_{2} \mathrm{O}_{2}$, after which incubated for 10-30minutes. Sections were washed in PBS buffer for 5minutes twice. 2 drops of primary mouse monoclonal antibodies included anti-HBME-1 antibody and anti-TPO antibody (DBS, USA) was used and incubated for 60 minutes. Sections were washed with PBS twice. Sections were then incubated with the linked antibody for 30 minutes and again two washings with PBS were given. Enzyme conjugate was added and incubated for 30 minutes. Again, two washings with PBS were given. One tablet of DAB was dissolved in reagent and incubated on the sections for 6-8 minutes. All incubation was done in a moist chamber. Sections were washed in deionized water for 3 minutes. Hematoxylin counterstaining was done for 2-5 minutes and sections washed under tap water and then dehydrated in ascending concentration of alcohol. The clearing was done in xylene and sections mounted with DPX.

IHC Interpretation: Immunoreactive scoring system was used. The immunoreactive score (IRS) gives a range of $0-12$ as a product of multiplication between positive cells proportion score (0-4) and staining intensity score $(0-3)$. The staining intensity was graded as 0 (no staining), 1+ (mild staining), $2+$ (moderate staining), or $3+$ (intense staining), and the proportion of stained cells was scored as 0 (no staining), $1+(1-10 \%$ of cells), $2+(10-50 \%$ of cell), $3+(50-80 \%$ of cells $), 4+(>80 \%$ of cells $)$. Cases that showed IRS score of $>3$, were scored as positive for TPO or HBME-1. A positive result for HBME-1 was considered consistent with malignancy, and a negative result for TPO was considered
Consistent with malignancy [8].

Sensitivity and specificity were calculated for HBME1 and TPO individually. Sensitivity was defined as the number of carcinomas with positive results (positive HBME-1 or negative TPO) as a percentage of the total number of carcinomas. Specificity was defined as the number of benign lesions with negative results as a percentage of the total number of benign lesions.

Statistical analysis: Data was analyzed using SPSS Software Package, Version 22 (Trial) and appropriate parametric and non- parametric tests were employed according to the studied variable. The agreement between the two methods of sample preparation was assessed with respect to the histological characteristics and immunohistochemistry. $\mathrm{P}$ values $</$ or $=$ to 0.05 were considered to be statistically significant.

\section{Results}

Incidence of thyroid nodules was most common in the age group between $21-40$ years comprising about $58 \%(29 / 50)$ of cases followed by $41-60$ years comprising $32 \%(16 / 50)$. Thyroid nodules were more common among females comprising about $86 \%(43 / 50)$ cases in contrast to males, with a Female to male ratio of $6: 1$. It was observed that the maximum number of cases of benign thyroid lesions i.e. 13 cases $(65 \%)$ and also of the malignant thyroid lesion i.e. 16 cases $(61.5 \%)$ cases belonged to the age group of $21-40$ years [Table-1].

Table-1: Age-wise distribution of thyroid lesions $(n=50)$.

\begin{tabular}{|l|l|l|l|l|l|}
\hline \multicolumn{2}{|c|}{} & \multicolumn{2}{c|}{ Malignant } & \multicolumn{2}{c|}{ Benign } \\
\cline { 2 - 6 } \multicolumn{2}{|c|}{} & No. of cases & Percentage & No. of cases & Percentage \\
\hline \multirow{4}{*}{ Age Group } & $<20$ & 1 & $3.8 \%$ & 3 & $13 \%$ \\
\cline { 2 - 6 } & $21-40$ & 16 & $61.5 \%$ & 13 & $65 \%$ \\
\cline { 2 - 6 } & $41-60$ & 8 & $30.8 \%$ & 8 & $40 \%$ \\
\cline { 2 - 6 } & $>60$ & 1 & $3.8 \%$ & 0 & $0 \%$ \\
\hline Total & 26 & $100.0 \%$ & 24 & $100 \%$ \\
\hline
\end{tabular}

Table-2: Expression of HBME-1 in both benign and malignant thyroid lesions $(n=50)$.

\begin{tabular}{|c|c|c|c|c|c|}
\hline $\begin{array}{l}\text { Thyroid } \\
\text { lesions }\end{array}$ & $\begin{array}{l}\text { Thyroid } \\
\text { lesions }\end{array}$ & Present & Absent & Total & Value \\
\hline \multicolumn{2}{|c|}{$\begin{array}{l}\text { Benign ( } n=24) \text { [Goiter, } \\
\text { Graves, CLT, FA] }\end{array}$} & $4(16.66 \%)$ & $20(83.3 \%)$ & $24(100 \%)$ & \\
\hline \multirow{3}{*}{$\begin{array}{l}\text { Malignant } \\
(n=26)\end{array}$} & PTC & $15(78.95 \%)$ & $4(21 \%)$ & $19(100 \%)$ & \multirow{3}{*}{$\begin{array}{l}0.074 \\
\#\end{array}$} \\
\hline & FTC & $2(50 \%)$ & $2(50 \%)$ & $4(100 \%)$ & \\
\hline & MTC & $0(0 \%)$ & $3(100 \%)$ & $3(100 \%)$ & \\
\hline
\end{tabular}


* Statistical analysis of data was done by using the Chi-square test. P-value $>0.05$ was considered significant. \# statistically not significant.

Table-3: Expression of TPO in both benign and malignant thyroid lesions $(n=50)$

\begin{tabular}{|l|l|l|l|l|c|}
\hline $\begin{array}{c}\text { Thyroid } \\
\text { lesions }\end{array}$ & Thyroid lesions & Present & Absent & Total & $\begin{array}{c}\text { P- } \\
\text { Value }\end{array}$ \\
\hline Benign $(n=24)$ & $\begin{array}{l}\text { [Goiter, Graves, CLT, } \\
\text { FA] }\end{array}$ & $\begin{array}{l}22(91.7 \\
\%)\end{array}$ & $2(8.3 \%)$ & $\begin{array}{l}24(100 \\
\%)\end{array}$ & \\
\hline $\begin{array}{l}\text { Malignant } \\
(n=26)\end{array}$ & PTC & $\begin{array}{l}7(36.84 \\
\%)\end{array}$ & $\begin{array}{l}12(63.16 \\
\%)\end{array}$ & $\begin{array}{l}19(100 \\
\%)\end{array}$ & \multirow{2}{*}{$0.070 \#$} \\
\cline { 2 - 5 } & FTC & $4(100 \%)$ & $0(0 \%)$ & $4(100 \%)$ & $0.462 \#$ \\
\cline { 2 - 5 } & MTC & $2(66.6 \%$ & $1(33.3 \%)$ & $3(100 \%)$ & \\
\hline
\end{tabular}

* Statistical analysis of data was done by using the Chi-square test. P-value $>0.05$ was considered significant. \# statistically not significant.

The cell membrane marker, HBME-1 expression (a marker for malignancy) was found absent in $83.3 \%$ cases of Benign thyroid lesions. Positive expression of HBME-1 in Malignant thyroid lesions was $78.95 \%$, $50 \%$ and $0 \%$ in PTC, FTC, and MTC respectively, whereas there was no expression of the marker in $21 \%, 50 \%$ and $100 \%$ cases of PTC, FTC and MTC respectively (Fig.1,2,3). Thus, depicting that overall, neoplastic thyroid lesions showed predominantly positive staining with HBME-1 [Table-2]. Cytoplasmic marker TPO showed positive expression in $91.7 \%$ of cases of benign thyroid lesions. In malignant thyroid lesions, negative expression of TPO (taking negative expression of TPO as a marker for malignancy) was shown by $63.16 \%, 0 \%$ and $33.33 \%$ cases of PTC, FTC, and MTC respectively. [Table-3] (Figure1, 2, 3)

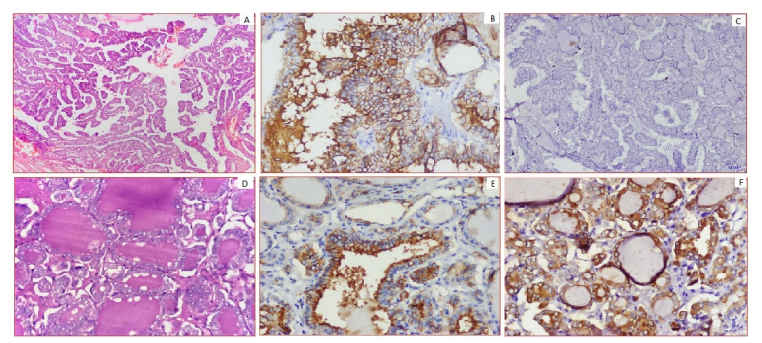

Fig-1; A-C: Photomicrograph showing classical PTC [A]- $H$ and $E$ stained section (200X). [B]HBME-1 Immunostaining showing strong membranous positivity (IHC; 400x). [C] TPO showing negative immunostaining (IHC; 200X). D-F: Photomicrograph showing PTCFOLLICULAR VARIANT [D]- $H$ and $E$ stained section (400X). [E]- HBME-1 Immunostaining
Showing strong membranous and few cytoplasmic positivity (IHC; 400X). [F]- TPO immunostaining showing moderate-intensity cytoplasmic positivity (IHC; 200X).

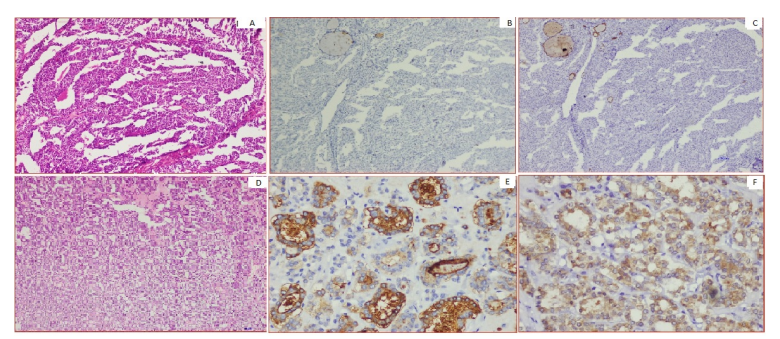

Fig-2; A-C: Photomicrograph showing MTC [A]$H$ and $E$ stained section (200X). [B]- HBME-1 showing negative immunostaining (IHC; 200x). [C] TPO showing negative immunostaining (IHC; 200X).

D-F: Photomicrograph showing FTC [D]- H and E stained section (200X). [E]- HBME-1 Immunostaining showing membranous positivity (IHC; 200X). [F]- TPO immunostaining showing mild intensity cytoplasmic positivity (IHC; 200X).

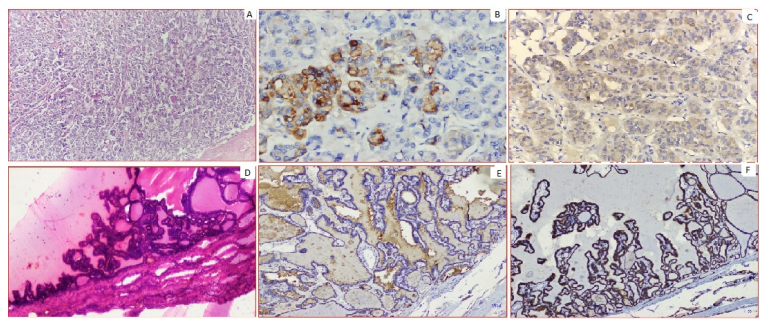

Fig-3: A-C: Photomicrograph showing Follicular Adenoma [A]- $H$ and $E$ stained section (200X). [B]- Showing moderateintensity HBME-1 immunostaining (IHC; 200x). [C] TPO showing mild cytoplasmic immunostaining (IHC; 200X). D-F: Photomicrograph showing Colloid Goiter with papillary hyperplasia [D]- $H$ and $E$ stained section (200X). [E]- HBME-1 showing negative immunostaining (IHC; 200X). [F]- TPO immunostaining showing strong cytoplasmic positivity (IHC; 200X).

\section{Discussion}

Thyroid cancer is one of the most common malignant tumors of the endocrine system, with a dramatically increased incidence [9]. The most frequent type of thyroid malignancy is PTC, which comprises $90 \%$ of all thyroid cancers [1]. Thus, it is 
Critical to accurately diagnose PTC. When the diagnosis of PTC is difficult to distinguish using histological criteria immunohistochemistry techniques are useful. According to the World Health Organization classification, PTC may be histologically classified into 14 variants based on histological structure, tumor size, cell shape, cell type, and extracellular matrix, including papillary microcarcinoma, encapsulated variant, follicular variant, diffuse sclerosing, tall cell, columnar, cribriform morular, hobnail, solid/trabecular, oncocytic, spindle cell variant, clear cell, warthin like and papillary thyroid carcinoma with fibromatosis/fasciitis like stroma variant. However, due to the paucity of diagnostic nuclear features; it is often difficult to distinguish papillary thyroid carcinoma from its architectural and nuclear mimics in thyroid lesions. In this condition, molecular alterations in thyroid cancer, such as TPO, and HBME-1, may help to differentiate papillary thyroid cancers from other thyroid lesions [7]. For example, one of the most frequent difficulties in thyroid pathology is differentiating the follicular variant of PTC from follicular adenoma. The inter observable variability in this area is well recognized. The differentiation is critical for the treatment and longterm management of the tumors. Despite the universal recognition of this variant, it is clearly lacking the minimal histological definition of the follicular variant of PTC. Unfortunately, the immune profile of follicular derived lesions and neoplasms show some overlap and to the best of our knowledge, no single marker or even panel is $100 \%$ sensitive and $100 \%$ specific for PTC. Papillary thyroid carcinoma is common in persons of all ages, with a peak age range between 20-60years $[10,11,12,13]$. In the present study age of the patient diagnosed with papillary thyroid cancer ranged from 21-40 years. Papillary thyroid carcinoma most commonly affects women [13]. Our results on gender distribution showed that the female population predominated comprising about $86 \%(n=43)$ of cases in contrast to males with Female to male ratio being $6: 1$. HBME-1 targets an unknown antigen of mesothelial microvilli and is abnormally expressed in thyroid malignancies, showing cytoplasmic location with membrane accentuation [14]. HBME-1 is highly expressed in PTC including both classical and follicular variant and FTC with little or no expression in MCT and ATC $[15,16]$. It has been noticed that HBME-1 is not expressed in benign thyroid lesion but expressed in malignant thyroid cancer, thus making HBME-1 the
Most specific and preferred marker for thyroid cancer and may be useful in differentiating malignant thyroid cancer from the benign tumor [17]. In the present study, when assessing the diagnostic performance of HBME-1 as a single protein marker, the sensitivity was $78.95 \%$ and $50 \%$ in PTC and FTC respectively. HBME-1 consistently showed negative staining in MTC. Whereas, in benign thyroid lesions HBME-1 positivity was seen in $16 \%$ of cases, showing only weak intensity of staining, with a specificity of $83.3 \%$. In accordance with the present study, previous studies have demonstrated that a high rate of HBME-1-positivity, by immunohistochemistry, was observed in malignant thyroid tissues mostly PTC followed by FTC along with reduced positivity for benign thyroid lesions and HBME-1 was a sensitive marker for PTCs [18-22]. Further, in variation to the present study, a study conducted by Zhu $X$ et al [23] showed HBME-1 sensitivity for PTC was $95.5 \%$. This variation could be due to the presence of the large number of cases taken for the study (155 cases of PTC), in comparison with the present study consisting of a total of 50 cases, out of which 19 cases were of PTC. Thyroid peroxidase is a thyroidspecific enzyme that catalyzes iodide oxidation, thyroglobulin iodination and iodothyronine coupling [5]. Reduced activity or expression of TPO impairs thyroid follicular cell function by reducing iodide trapping and impairing thyroid hormone synthesis [5]. Moreover, TPO has also been shown to suppress thyroid cell proliferation and its loss has been associated with thyroid carcinogenesis. In the present study, it was reported that TPO was expressed in $91.7 \%$ of cases of benign thyroid lesions. TPO, a marker whose expression is reduced or absent in thyroid carcinoma, revealed a negative, "malignant" profile, in $63.16 \%$ cases of PTC, and $33.33 \%$ case of MCT. By using a positive stain for TPO as negative or not suggestive of malignancy TPO had a specificity of $91.7 \%$. In accordance to the present study, previous studies reported TPO, to be a practical marker in terms of diagnostic utility in distinguishing non-malignant thyroid nodules from thyroid malignancy and showed that TPO had a 50\% sensitivity for the diagnosis of PTC, suggesting that it should be combined with other markers [24,25]. Another study by Acrolia $\mathrm{V}$ et al. also reported that TPO expression was higher in benign thyroid lesions than malignant thyroid lesions as seen in the present study [26]. In variation to the present study, the results for TPO from the present study were quite different from what has been reported in a previous study showing the sensitivity of TPO 
Staining for diagnosis of malignancy as $100 \%$ [27]. The sensitivity in the present study was only $63.3 \%$ for PTC and $33.33 \%$ for follicular carcinomas. The reasons for these discrepancies are unclear. These results do not reflect nonspecific staining, because there were cases of papillary and follicular carcinomas that clearly showed negative TPO staining. Morphological diagnosis methods such as immunohistochemistry and cytology of fine-needle aspiration biopsy play an important role in providing a definitive diagnosis and therapeutic guidance. Testing for expression of HBME-1 has been shown to improve the diagnostic accuracy for thyroid malignant nodules $[28,29]$. The combination of HBME-1 and TPO may contribute to an accurate diagnosis of thyroid papillary carcinoma by fineneedle aspiration biopsy.

Limitations: In comparison to other studies, a few limitations in the present study were present. It was observed that sensitivity to both the markers in differentiating papillary thyroid carcinoma from another thyroid neoplasm could be better studied with a greater number of cases. Rather, than using a single marker, a panel of markers will be more helpful in reaching diagnosis e.g. galectin-3, CD56, CK19, along with HBME-1 and TPO. No standard IHC scoring system is available for these markers. Percentage and intensity staining scores were calculated manually so interobserver variations can be the reason for discrepancies.

\section{Conclusion}

In conclusion, due to the overlapping histological features, diagnostic problems in thyroid pathology still remains. HBME-1 due to its high sensitivity can significantly be used as a marker of identification for papillary thyroid carcinomas while TPO though helps in differentiating benign from malignant thyroid carcinomas but cannot be used alone. Therefore, a panel of IHC markers is always recommended for the diagnosis of malignant thyroid neoplasms.

\section{What does the study add to the existing knowledge}

Using the combination of HBME-1 and TPO is useful in differentiating benign from malignant thyroid neoplasms. However, a panel of markers more specific for papillary thyroid neoplasm including Galectin 3, CD56, CK19 along with HBME-1 and TPO will be more helpful in accurately diagnosing PTC from another thyroid neoplasm.'

\section{Authors' Contributions}

Dr. Permeet Kaur Bagga: Conduct of study by the selection of cases to be included in the study, histopathological and immunohistochemistry interpretations, compilation of data.

Dr. Vasundhara Singh: Collection of material, interpretation of immunohistochemistry, a compilation of data and drafting of the manuscript.

Dr. Bikramjeet Singh: Provided the relevant material and data.

Dr. Jaideep: Proofread and approved the final manuscript.

\section{Reference}

01. Hodgson NC, Button J, Solorzano CC. Thyroid cancer is the incidence still increasing?. Ann Surg Oncol. 2004;11(12)1093-1097. doi: [Article] [Crossref]

02. Pellegriti G, Frasca F, Regalbuto C, Squatrito S, Vigneri R. Worldwide increasing incidence of thyroid cancer update on epidemiology and risk factors. J Cancer Epidemiol. 2013.

doi: [Article] [Crossref]

03. Dinets $A$, Hulchiy $M$, Sofiadis $A$, Ghaderi $M$, Höög $A$, Larsson $C$, et al. Clinical, genetic, and immunohistochemical characterization of 70 Ukrainian adult cases with post-Chornobyl papillary thyroid carcinoma. Eur J Endocrinol. 2012;166(6)1049-1060.

doi: [Article] [Crossref]

04. Nguyen QT, Lee EJ, Huang MG, Park YI, Khullar A, Plodkowski RA. Diagnosis and treatment of patients with thyroid cancer. Am Health Drug Benefits. 2015;8(1)30.

[Crossref]

05. Stathathos N, Ringel. Molecular markers of thyroid nodules. In Adv Mol Cell Endocrinol. 2006; $4 ; 19-34$. doi: [Article] [Crossref]

06. De Micco C, Vasko V, Garcia S, Zoro P, Denizot A, Henry JF. Fine-needle aspiration of thyroid follicular neoplasm diagnostic use of thyroid peroxidase immunocytochemistry with monoclonal antibody $47 . \quad$ Surg. 1994;116(6)1031-1035.

[Crossref] 
07. Wu G, Wang J, Zhou Z, Li T, Tang F. Combined staining for immunohistochemical markers in the diagnosis of papillary thyroid carcinoma improvement in the sensitivity or specificity?. J Int Med Res. 2013;41(4)975-983.

doi: [Article] [Crossref]

08. Fedchenko N, Reifenrath J. Different approaches for interpretation and reporting of immunohistochemistry analysis results in the bone tissue - a review. Diagn Pathol. $2014 ; 9 ; 221$.

doi: [Article] [Crossref]

09. Kilfoy BA, Zheng T, Holford TR, Han X, Ward MH, Sjodin $A$, et al. International patterns and trends in thyroid cancer incidence, 1973-2002. Cancer Causes Control. 2009; 20(5)525-531. doi: [Article] [Crossref]

10. Papotti M, Rodriguez J, De Pompa R, Bartolazzi A, Rosai J. Galectin-3 and HBME-1 expression in well-differentiated thyroid tumors with follicular architecture of uncertain malignant potential. Mod Pathol. 2005;18(4)541-546.

doi: [Article] [Crossref]

11. Al Zaher N, Al Salam S, El Teraifi H. Thyroid carcinoma in the United Arab Emirates perspectives and experience of a tertiary care hospital. Hematol Oncol Stem Cell Ther. 2008;1(1)14-21.

doi: [Article] [Crossref]

12. Sumana BS, Shashidhar S, Shivarudrappa AS. Galectin-3 Immunohistochemical Expression in Thyroid Neoplasms. J Clin Diagn Res. 2015;9(11)EC07-EC11. doi: [Article] [Crossref]

13. Dwivedi SS, Khandeparkar SG, Joshi AR, Kulkarni MM, Bhayekar P, Jadhav A et al. Study of immunohistochemical markers (CK-19, CD$56, \mathrm{Ki}-67, \mathrm{p} 53$ ) in differentiating benign and malignant solitary thyroid nodules with special reference to papillary thyroid carcinomas. J Clin Diagn Res. 2016;10(12)EC14-EC19. doi: [Article] [Crossref]

14. Sack MJ, Astengo-Osuna $C$, Lin BT, Battifora $H$, LiVolsi VA. HBME-1 immunostaining in thyroid fine-needle aspirations a useful marker in the diagnosis of carcinoma. Mod path. 1997; 10(7)668-674.

[Crossref]
15. Miettinen $M$, Kärkkäinen $P$. Differential reactivity of HBME-1 and CD15 antibodies in benign and malignant thyroid tumours. Virchows Archiv. 1996;429(4-5)213-219.

doi: [Article] [Crossref]

16. Cheung, C, Ezzat S, Freeman JL, Rosen BI, Asa SL. Immunohistochemical Diagnosis of Papillary Thyroid Carcinoma. Mod Pathol. 2001;14(4)33842.

doi: [Article] [Crossref]

17. De Micco C, Savchenko V, Giorgi R, Sebag F, Henry JF. Utility of malignancy markers in fineneedle aspiration cytology of thyroid nodules co -mparison of Hector Battifora mesothelial anti gen-1, thyroid peroxidase and dipeptidyl amino -peptidase IV. Br J Cancer. 2008;98(4)818-823. doi: [Article] [Crossref]

18. Nga ME, Lim GS, Soh $\mathrm{CH}$, Kumarasinghe MP. HBME-1 and CK19 are highly discriminatory in the cytological diagnosis of papillary thyroid carcinoma. Diagn Cytopathol. 2008;36(8)550556.

doi: [Article] [Crossref]

19. Mase $T$, Funahashi $H$, Koshikawa $T$, Imai $T$, Nara $Y$, Tanaka $Y$, et al. HBME-1 immunostaining in thyroid tumors especially in follicular neoplasm. Endocr J. 2003;50(2)173-177.

doi: [Article] [Crossref]

20. Liu Z, Xun X, Wang Y, Mei L, He L, Zeng W, et al. MRI and ultrasonography detection of cervical lymph node metastases in differentiated thyroid carcinoma before reoperation. Am J Transl Res. 2014;6(2)147-154.

[Crossref]

21. De Matos PS, Ferreira AP, de Oliveira Facuri $F$, Assumpcao LV, Metze $\mathrm{K}$, et al. Usefulness of HBME-1, cytokeratin 19 and galectin-3 immunostaining in the diagnosis of thyroid mali -gnancy. Histopathology. 2005;47(4)391-401. doi: [Article] [Crossref]

22. Palo S, Biligi DS. Differential diagnostic significance of HBME-1, CK19 and S100 in various thyroid lesions. Malays J Pathol. 2017;39(1)55-67.

[Crossref]

23. Zhu X, Sun T, Lu H, Zhou X, Lu Y, Cai X et al. Diagnostic significance of CK19, RET, galectin-3 and HBME-1 expression for papillary thyroid carcinoma. J Clin Pathol. 2010;63(9)786-789. doi: [Article] [Crossref] 
24. Griffith OL, Melck A, Jones SJ, Wiseman SM. Meta-analysis and meta-review of thyroid cancer gene expression profiling studies identifies important diagnostic biomarkers. J Clin Oncol. 2006;24(31)5043-5051. doi: [Article] [Crossref]

25. Weber KB, Shroyer KR, Heinz DE, Nawaz S, Said MS, Haugen BR. The use of a combination of galectin-3 and thyroid peroxidase for the diagnosis and prognosis of thyroid cancer. Am J Clin Pathol. 2004;122(4)524-31. doi: [Article] [Crossref]

26. Arcolia V, Journe F, Renaud F, Leteurtre E, Gabius $H J$, Remmelink $M$, et al. Combination of galectin-3, CK19 and HBME-1 immunostaining improves the diagnosis of thyroid cancer. Onco lett. $2017 ; 14(4) 4183-4189$.

doi: [Article] [Crossref]
28. Henry JF, Denizot A, Porcelli A, Villafane M, ZoroP, Garcia $S$ et al. Thyroperoxidase immunodetection for the diagnosis of malignancy on fine-needle aspiration of thyroid nodules. World J Surg. 1994;18(4)529-534. doi: [Article] [Crossref]

29. Schmitt AC, Cohen C, Siddiqui MT. Paired box gene 8, HBME-1, and cytokeratin 19 expression in preoperative fine-needle aspiration of papillary thyroid carcinoma diagnostic utility. Cancer Cytopathol. 2010;118(4)196-202. doi: [Article] [Crossref]

30. Raggio E, Camandona $M$, Solerio D, Martino $P$, Franchello $A$, Orlandi $F$, et al. The diagnostic accuracy of the immunocytochemical markers in the pre-operative evaluation of follicular thyroid lesions. J Endocrinol Invest. 2010;33;378-381. doi: [Article] [Crossref] 